\title{
A CHARACTERIZATION THEOREM FOR THE DISCRETE BEST MONOTONIC APPROXIMATION PROBLEM
}

\author{
I. C. DEMETRIOU
}

\begin{abstract}
A characterization theorem is derived that motivates a procedure for generating discrete best monotonic approximations to $n$ sequential data values, when a strictly convex objective function is used in the calculation. The procedure is highly useful in the discrete nonlinear optimization calculation that produces the best piecewise monotonic approximations to the data.
\end{abstract}

\section{INTRODUCTION}

Let $f(x)$ be a smooth function of a single variable defined on the interval $[a, b]$ of the real line, and let $n$ be a positive integer. The function is measured on the finite mesh of data points $a=x_{1}<x_{2}<\cdots<x_{n}=b$; the measurements $\left\{\phi_{i} ; i=1,2, \ldots, n\right\}$ contain random errors $\left\{e_{i} ; i=1,2, \ldots, n\right\}$ so that $\left\{\phi_{i}=f\left(x_{i}\right)+e_{i} ; i=1,2, \ldots, n\right\}$. We regard the measurements as a vector $\phi$ in $\mathbb{R}^{n}$.

Let $S \subset \mathbb{R}^{n}$ be the set of vectors $\mathbf{y}$ in $\mathbb{R}^{n}$ whose components satisfy the monotonicity conditions

$$
y_{1} \leq y_{2} \leq \cdots \leq y_{n} .
$$

For any $\phi$ in $\mathbb{R}^{n}$, a vector $\mathbf{y}^{*}$ in $S$ is a best monotonically increasing approximation to $\phi$ if it minimizes a function of the form

$$
\Phi\left(y_{1}, y_{2}, \ldots, y_{n}\right)=\sum_{i=1}^{n} h\left(\phi_{i}-y_{i}\right)
$$

subject to $\mathbf{y} \in S$, where $h$ is a continuous strictly convex function of one variable whose least value is $h(0)$ and $h(\theta) \rightarrow \infty$, as $|\theta| \rightarrow \infty$. From the assumptions on $h$ it follows that, for all $i$,

(a) $\eta>\tau \geq \phi_{i}$ implies $h\left(\phi_{i}-\eta\right)>h\left(\phi_{i}-\tau\right)$,

(b) $\eta<\tau \leq \phi_{i}$ implies $h\left(\phi_{i}-\eta\right)>h\left(\phi_{i}-\tau\right)$,

and for any $\sigma$

(c) $h\left(\phi_{i}-\sigma\right)$ is a minimum if and only if $\sigma=\phi_{i}$.

Received November 15, 1988; revised July 11, 1989.

1980 Mathematics Subject Classification (1985 Revision). Primary 41A29, 65D10.

Key words and phrases. Data smoothing, monotonic.

This work has been carried out at the Department of Applied Mathematics and Theoretical Physics (Silver St.) of the University of Cambridge, United Kingdom.

(C) 1990 American Mathematical Society $0025-5718 / 90 \$ 1.00+\$ .25$ per page 
There exists a unique best monotonically increasing approximation to $\phi$ (Theorem 1 in §2) that can be efficiently derived by a method in [1].

In [3] we let $h(\theta)=\theta^{2}$ in (1.2) and develop an algorithm that calculates the numbers

$$
\alpha_{j}=\min _{y_{1} \leq y_{2} \leq \cdots \leq y_{j}} \sum_{i=1}^{j} h\left(\phi_{i}-y_{i}\right), \quad j=1,2, \ldots, n,
$$

in $O(n)$ computer operations. The algorithm is highly useful in the discrete nonlinear smoothing calculation that minimizes $(1.2)$ with $h(\theta)=\theta^{2}$, subject to a prescribed number of sign changes, say $q$, in the sequence of the first divided differences of the smoothed values. This calculation is carried out by a recursive technique $[3,2]$ in $O\left(n^{2}+q \cdot n \cdot \log _{2} n\right)$ computer operations by employing a large number of minimization calculations of the form (1.3).

The purpose of this article is to show that the algorithm mentioned above, after some obvious modifications, is applicable for $h$ any strictly convex function as defined immediately after formula (1.2). A characterization theorem is derived that is proved by arguments more general than those in [3].

The paper is organized as follows. The existence and uniqueness of a best monotonically increasing approximation to $\phi$ is asserted in Theorem 1 of $\S 2$. Then Lemma 1 shows that one can calculate the best monotonically increasing approximation to $\phi$ by seeking the intervals where its components have different constant values. The intervals may be found using the remarkable property that any conditions which are satisfied as equalities by the approximation that occurs in the definition of $\alpha_{j}$ are also satisfied as equalities by the one in $\alpha_{j+1}$; cf. Lemma 3 and Theorem 2. Hence, the quantities (1.3) can be derived by a method similar to [3] in exactly $n-1$ iterations.

\section{THE BEST MONOTONICALLY INCREASING APPROXIMATION}

The existence of a best approximation from $S$ to $\phi$ is established by Theorem 1 , which is a consequence of Weierstrass' theorem. The uniqueness follows from the strict convexity of the function (1.2).

Theorem 1. Let the function $\Phi$ and the set $S$ be as defined in $\S 1$. Then there exists a unique best monotonically increasing approximation from $S$ to $\phi \in \mathbb{R}^{n}$.

Proof. Since $\Phi$ is continuous and $\Phi(\mathbf{y}) \rightarrow \infty$ as $\|\mathbf{y}\|_{\infty} \rightarrow \infty$, the set $V=$ $\{\mathbf{y}: \Phi(\mathbf{y}) \leq \Phi(\mathbf{0})\}$ is closed and bounded; here $\mathbf{0} \in V$. Since $S$ is closed and $\mathbf{0} \in S$, the set $V \cap S$ is closed, bounded and nonempty. Hence, from the continuity of $\Phi(\mathbf{y})$ for $\mathbf{y} \in V \cap S$, there exists a finite value $u^{*}$ and a vector $\mathbf{y}^{*} \in V \cap S$ such that $\Phi\left(\mathbf{y}^{*}\right)=u^{*}=\min _{\mathbf{y} \in \operatorname{l} \cap S} \Phi(\mathbf{y})$. As $\inf _{\mathbf{y} \in S} \Phi(\mathbf{y})=$ $\min _{\mathbf{y} \in V \cap S} \Phi(\mathbf{y})$, it follows that $\Phi\left(\mathbf{y}^{*}\right)=u^{*}=\inf _{\mathbf{y} \in S} \Phi(\mathbf{y})$. Thus $\mathbf{y}^{*}$ minimizes $\Phi$ on $S$. Further, since $\Phi$ is strictly convex, it can be shown by well-known arguments that $\mathbf{y}^{*}$ is unique. 
A useful property of the best monotonically increasing approximation is stated in the following lemma. It shows that any condition $y_{i} \leq y_{i+1}$ that is satisfied by $\mathbf{y}^{*}$ in the form $y_{i}^{*}<y_{i+1}^{*}$ provides some separability in the calculation of $\mathbf{y}^{*}$.

Lemma 1. Let $h$ be the function defined in $\S 1$, let $\mathbf{y}^{*}$ be the member of $\mathbf{y} \in S$ that minimizes the function (1.2), and let $s$ be any integer in $[1, n-1]$ such that $y_{s}^{*}<y_{s+1}^{*}$. Then $\left\{y_{i}^{*} ; i=1,2, \ldots, s\right\}$ and $\left\{y_{i}^{*} ; i=s+1, s+2, \ldots, n\right\}$ are the best monotonically increasing approximations to $\left\{\phi_{i} ; i=1,2, \ldots, s\right\}$ and $\left\{\phi_{i} ; i=s+1, s+2, \ldots, n\right\}$, respectively.

Proof. We assume the contrary, and define $\hat{\mathbf{y}} \in \mathbb{R}^{n}$ to be the vector that minimizes (1.2) subject to the conditions $y_{1} \leq y_{2} \leq \cdots \leq y_{s}$ and $y_{s+1} \leq y_{s+2} \leq$ $\cdots \leq y_{n}$. If $\hat{\mathbf{y}}$ happens to satisfy the inequality $\hat{y}_{s} \leq \hat{y}_{s+1}$, then $\hat{\mathbf{y}} \in S$ and, by assumption, we obtain $\Phi(\hat{\mathbf{y}})<\Phi\left(\mathbf{y}^{*}\right)$, a contradiction to the optimality of $\mathbf{y}^{*}$. If $\hat{y}_{s}>\hat{y}_{s+1}$, let $\tilde{\mathbf{y}}(\theta)=\theta \mathbf{y}^{*}+(1-\theta) \hat{\mathbf{y}}, \theta \in[0,1]$. As $y_{s}^{*}<y_{s+1}^{*}$, there exists a $\theta_{0} \in(0,1)$ such that $\tilde{\mathbf{y}}(\theta) \in S$ for $\theta \in\left[\theta_{0}, 1\right)$. Hence by the convexity of $\Phi$ and the initial assumption, we obtain

$$
\Phi(\tilde{\mathbf{y}}(\theta)) \leq \theta \Phi\left(\mathbf{y}^{*}\right)+(1-\theta) \Phi(\hat{\mathbf{y}})<\Phi\left(\mathbf{y}^{*}\right), \quad \theta \in\left[\theta_{0}, 1\right),
$$

again a contradiction. The assertion of the lemma follows.

Lemma 1 implies that, in order to calculate $\mathbf{y}^{*}$, we can equivalently seek the conditions in (1.1) which are satisfied as equalities in $\mathbf{y}^{*}$. Theorem 2 below provides the basis for determining these conditions. To prove Theorem 2 , we need two lemmas, which are stated after the following definition. If $s$ and $t$ are any integers such that $1 \leq s \leq t \leq n$, define $\eta_{s t}$ to be the value of $\eta$ that minimizes the expression

$$
\Phi_{s t}(\eta)=\sum_{i=s}^{t} h\left(\phi_{i}-\eta\right) .
$$

Since the function $h$ in $(2.1)$ is strictly convex, $\eta_{s t}$ is unique.

Lemma 2. Let the integers $s, t$ be given such that $1 \leq s \leq t \leq n$ and let $\left\{y_{i}^{*} ; i=s, s+1, \ldots, t\right\}$ be the best monotonically increasing approximation to $\left\{\phi_{i} ; i=s, s+1, \ldots, t\right\}$. If $y_{s}^{*}=y_{s+1}^{*}=\cdots=y_{t}^{*}=\eta_{s t}$, then

$$
\eta_{s l} \geq \eta_{s t} \geq \eta_{l+1, t} \text { for } s \leq l<t \text {. }
$$

Proof. The inequality $\eta_{s l}<\eta_{l+1, t}$ cannot hold because otherwise we can replace each member of the sequence $\left\{y_{i}^{*} ; i=s, s+1, \ldots, l\right\}$ by $\eta_{s l}$ and each member of the sequence $\left\{y_{i}^{*} ; i=l+1, l+2, \ldots, t\right\}$ by $\eta_{l+1, t}$, which maintains monotonicity but reduces the value of $\sum_{i=s}^{t} h\left(\phi_{i}-y_{i}\right)$. A contradiction is derived, and the inequality $\eta_{s l} \geq \eta_{l+1, t}$ follows.

In order to prove (2.2), we first suppose that $\eta_{l+1, t}>\eta_{s t}$. Then $\eta_{s l} \geq \eta_{l+1, t}>$ $\eta_{s t}$. From this, and using a reasoning that is based on the strict convexity of $h$ 
and (2.1), we obtain

$$
\begin{aligned}
\Phi_{s t}\left(\eta_{s t}\right)= & \Phi_{s l}\left(\eta_{s t}\right)+\Phi_{l+1, t}\left(\eta_{s t}\right) \\
& >\Phi_{s l}\left(\eta_{l+1, t}\right)+\Phi_{l+1, t}\left(\eta_{l+1, t}\right)=\Phi_{s t}\left(\eta_{l+1, t}\right),
\end{aligned}
$$

a contradiction to the definition of $\eta_{s t}$. It follows that $\eta_{l+1, t} \leq \eta_{s t}$. By similar arguments we derive $\eta_{s t} \leq \eta_{s l}$. The last two inequalities complete the proof of the lemma.

Lemma 3. Let $\mathbf{y}^{*}$ be the member of $\mathbf{y} \in S$ that minimizes (1.2), let $\hat{I}$ be any set of integers such that $\hat{I} \subset\{1,2, \ldots, n-1\}$ and let $\hat{\mathbf{y}}$ be the vector that minimizes (1.2) subject to the conditions $\left\{y_{i} \leq y_{i+1} ; i \in \hat{I}\right\}$. Then, the inequalities

$$
\hat{y}_{1} \geq y_{1}^{*} \text { and } \hat{y}_{n} \leq y_{n}^{*}
$$

hold. Further, if $j$ is an integer in $[1, n-1]$ such that $y_{j}^{*}<y_{j+1}^{*}$, then

$$
\hat{y}_{j} \leq y_{j}^{*} \text { and } y_{j+1}^{*} \leq \hat{y}_{j+1} \text {. }
$$

Proof. Assume that $1 \in \hat{I}$, because otherwise $\hat{y}_{1}=\phi_{1}$ and the first inequality in (2.3) follows from the fact that $\phi_{1} \geq y_{1}^{*}$. Let $s-1$ be the greatest integer in $\hat{I}$ such that

$$
\hat{y}_{1}=\hat{y}_{2}=\cdots=\hat{y}_{s},
$$

which, in view of the comment following Lemma 1 , implies that $\left\{\hat{y}_{i} ; i=\right.$ $1,2, \ldots, s\}$ is the best monotonically increasing approximation to the first $s$ data. If $y_{s}^{*}<y_{s+1}^{*}$, then $\hat{y}_{1}=y_{1}^{*}$ by uniqueness arguments; otherwise, we assume that $\hat{y}_{1}<y_{1}^{*}$, which, owing to the conditions satisfied by $\mathbf{y}^{*}$ and (2.5), implies that $\hat{y}_{s}<y_{s}^{*}$. Hence, we can replace the first $s$ components of $\mathbf{y}^{*}$ by the first $s$ components of $\hat{\mathbf{y}}$, which maintains monotonicity but reduces the value of $\Phi(\mathbf{y})$, for we have ruled out the possibility that $\left\{y_{i}^{*} ; i=1,2, \ldots, s\right\}$ is the best monotonically increasing approximation to the first $s$ data. Therefore, $\hat{y}_{1} \geq y_{1}^{*}$ follows, and $\hat{y}_{n} \leq y_{n}^{*}$ may be shown similarly.

In order to show the relations $(2.4)$, we assume that $\hat{y}_{j}=\hat{y}_{j+1}$, because otherwise these relations follow from the first part of the lemma. Let $\hat{y}_{j}=\hat{y}_{j+1}>$ $y_{j+1}^{*}$, let $m(j)$ and $g(j)$ be the smallest and the largest integer, respectively, in $\hat{I}$ such that $\left\{\hat{y}_{i}=\hat{y}_{j} ; i \in[m(j), g(j)]\right\}$, and let $k$ be the smallest integer in the set $\left\{i ; y_{i}^{*}<y_{i+1}^{*}, i \in[m(j), j]\right\}$. Then $y_{k}^{*}=\eta_{l k}$ for some integer $l$ in $[1, m(j)]$. From this, and by Lemma 2, we obtain $\eta_{m(j), k} \geq \eta_{m(j), g(j)}$ and $\eta_{m(j), k} \leq y_{k}^{*}$. Thus,

$$
\eta_{m(j), k} \leq y_{k}^{*} \leq y_{j}^{*}<\hat{y}_{j}=\eta_{m(j), g(j)} \leq \eta_{m(j), k},
$$

a contradiction. We similarly derive a contradiction if we assume that $y_{j}^{*} \leq$ $\hat{y}_{j}=\hat{y}_{j+1} \leq y_{j+1}^{*}$ or $y_{j}^{*}>\hat{y}_{j}=\hat{y}_{j+1}$. Hence, the assumption $\hat{y}_{j}=\hat{y}_{j+1}$ must be false. Thus $\hat{y}_{j} \neq \hat{y}_{j+1}$ and (2.4) follows from Lemma 1 and the first part of this lemma.

The characterization theorem for the best monotonically increasing approximation to $\phi$ now follows. 
Theorem 2. Let $\mathbf{y}^{*}$ be the member of $\mathbf{y} \in S$ that minimizes (1.2), let $\hat{I}$ be any subset of $\{1,2, \ldots, n-1\}$ and let $\hat{\mathbf{y}}$ be the vector that minimizes $\Phi(\mathbf{y})$ subject to $\left\{y_{i} \leq y_{i+1} ; i \in \hat{I}\right\}$. If $j$ is any integer in $[1, n-1]$ such that $\hat{y}_{j} \geq \hat{y}_{j+1}$, then $y_{j}^{*}=y_{j+1}^{*}$.

Proof. If $y_{j}^{*}<y_{j+1}^{*}$, then in view of inequalities (2.4) and the definition of $\hat{\mathbf{y}}$ we obtain $\hat{y}_{j}<\hat{y}_{j+1}$, which contradicts the definition of $j$. Hence, the assumption made must be false and the equation $y_{j}^{*}=y_{j+1}^{*}$ follows. The proof of the theorem is complete.

Theorem 2 shows that if $\hat{\mathbf{y}}$ is the best approximation to $\phi$ subject to a subset of the monotonicity conditions (1.1), then any condition violated by $\hat{\mathbf{y}}$ is satisfied as an equality by the solution. Thus, the theorem allows the development of an iterative procedure that inserts one monotonicity condition at a time in $\hat{\mathbf{y}}$, and therefore terminates in exactly $n-1$ iterations as the process suggested in [3], giving the best monotonically increasing approximation to the data.

\section{ACKNOWLEDGMENTS}

Professor M. J. D. Powell of the University of Cambridge, U.K., suggested this topic and gave me much encouragement. I feel grateful to him. I am also grateful to Dr. J. A. Gregory of Brunel University and Dr. A. Iserles of Cambridge University for their constructive comments. I would also like to thank the referee for his useful suggestions that improved the paper.

I am in debt to the State Scholarship Foundation (IKY) of Greece for sponsoring this work as part of my Ph. D. Dissertation.

\section{BIBLIOGRAPHY}

1. C. P. Cullinan and M. J. D. Powell, Data smoothing by divided differences, Numerical Analysis (Proceedings, Dundee 1981) (G. A. Watson, eds.), Springer-Verlag, New York, 1982.

2. I. C. Demetriou, Data smoothing by piecewise monotonic divided differences, $\mathrm{Ph}$. D. Dissertation, University of Cambridge, 1985.

3. I. C. Demetriou and M. J. D. Powell, Least squares smoothing of univariate data to achieve piecewise monotonicity, Report DAMTP 1989/NA9, University of Cambridge, UK (to appear in IMA J. Numer. Anal.). 